\title{
COMUNICAÇÃO
}

\section{CARACTERÍSTICAS FÍSICAS E QUÍMICAS DE CULTIVARES DE MILHO PARA PRODUÇÃO DE MINIMILHO}

\author{
RENZO GARCIA VON PINHO ${ }^{1}$ \\ GRACIELA SILVA CARVALHO ${ }^{2}$ \\ VICTOR DO NASCIMENTO RODRIGUES ${ }^{3}$ \\ JOELMA PEREIRA ${ }^{4}$
}

\begin{abstract}
RESUMO - Minimilho, também conhecido como "baby corn", é o nome dado à espiga jovem, em desenvolvimento, não fertilizada de uma planta de milho. Várias cultivares de milhos especiais, como doce, pipoca, e cultivares prolíficas de milho comum têm sido utilizadas para a produção do minimilho. Há escassez de informações no Brasil com referência ao manejo de produção do minimilho, bem como sobre o desempenho das cultivares, principalmente sobre tecnologia para processamento agroindustrial e constituição química do minimilho. Objetivou-se com este trabalho determinar características físicas e químicas de cultivares visando à produção de minimilho. O experimento foi instalado
\end{abstract}

em 10/02/2001 na área experimental do Departamento de Agricultura no campus da UFLA, em Lavras, MG. Para a determinação das características, foi retirada uma amostra das espigas de cada cultivar avaliada. Realizaram-se três colheitas, a intervalos de três dias, sendo a primeira três dias após a emissão dos estilosestigma. Foram determinados composição química, teores de açúcares, vitamina $\mathrm{C}$, compostos fenólicos e indicadores de qualidade. Os valores de umidade, de carboidratos, de proteína, de açúcares e de vitaminas das cultivares analisadas apresentaram-se similares aos comumente relatados na literatura. Os valores obtidos para cada característica variam em função da cultivar considerada.

TERMOS PARA INDEXAÇÃO: Zea mays, cultivares, minimilho, composição física, composição química.

\section{PHYSICS AND CHEMISTRY CHARACTERISTICS OF CORN CULTIVARS TO BABYCORN PRODUCTION}

\begin{abstract}
Baby corn is the name given to the young corn-cob when the ear has not been fertilized yet. Several sweet, popcorn and high yield cultivars selected from regular maize have been chosen for baby corn production. There is few information about baby corn production and performance in Brazil, its nutritional values, as well as its processing and storage techniques. The objective of this work was to determine physical and chemical parameters of baby corn. An experiment was established in the research area of the Agricultural Department of Federal University of Lavras (UFLA), at
\end{abstract}

Lavras, MG, Brazil. To determine physical and chemical characteres of baby corn a sample was obtained of each cultivar. Three baby corn harvesting were performed at 3-days interval with the first occurring 3 days from style-stigma emission (65 days after sowing). The aproximate chemical composition, the contents of sugar, vitamin $\mathrm{C}$, fenolics compound and indicators of the quality were determined. The physical and chemical characteres of baby corn were similar to those reported in the literature for normal corn.

INDEX TERMS: Zea mays, cultivars, baby corn, physical composition, chemical composition.

1. Engenheiro Agrônomo, DSc., Professor Adjunto do Departamento de Agricultura da UNIVERSIDADE FEDERAL DE LAVRAS/UFLA, Caixa Postal 37, 37200-000, Lavras, MG, renzo@ufla.br.

2. Engenheiro Agrônomo, MSc., graciela.silva@zipmail.com.br

3. Engenheiro Agrônomo, Mestrando no Departamento de Agricultura/UFLA, victor@ufla.br.

4. Engenheiro Agrônomo, DSc., Professora Adjunto do Departamento de Ciência dos Alimentos/UFLA, joelma@ufla.br. 
O minimilho, também conhecido como "baby corn", é o nome dado à espiga de milho jovem, em desenvolvimento, não fertilizada, ou ao sabugo jovem da espiga de uma planta de milho (GALINAT e LIN, 1988). As plantas são semelhantes às de milho normal $\mathrm{e}$ não são, como poderia ser assumido, plantas anãs (MILES e ZENS, 1998).

O minimilho é similar, na sua composição, a outras hortaliças, como a couve-flor, o tomate, a berinjela e o pepino. Em $100 \mathrm{~g}$ de amostra, o teor de proteína é de $1,90 \mathrm{~g}$; na couve-flor, $2,40 \mathrm{~g}$; na couve, $1,70 \mathrm{~g}$; no tomate e na berinjela, $1,00 \mathrm{~g}$; e no pepino, $0,60 \mathrm{~g}$. $\mathrm{O}$ minimilho possui cerca de $89,1 \%$ de umidade, $0,20 \%$ de gordura, $8,20 \%$ de carboidratos e $0,60 \%$ de cinzas. Cem gramas de minimilho contêm, em média, $86 \mathrm{mg}$ de fósforo, 0,1 mg de ferro, 64 UA de vitamina A, 0,05 $\mathrm{mg}$ de tiamina, 0,8 $\mathrm{mg}$ de riboflavina, 11,0 $\mathrm{mg}$ de ácido ascórbico e 0,3\% de niacina (YODPET, 1979).

A Tailândia é um dos principais paises produdores, sendo o maior exportador. Em 2000, a exportação de minimilho enlatado da Tailândia foi de 55.000 mil toneladas, correspondente a cerca de 42 milhões de dólares. Desses, $42,8 \%$ foram exportados para os EUA, $8,9 \%$ para a Austrália e $8,9 \%$ para o Japão (AETAKASANAWAN, 2001).

A exportação de minimilho in natura é menor do que a do enlatado. Em 2000, dos 4,3 milhões de dólares exportados pela Tailândia (que equivalem a 4.200 toneladas), $50,7 \%$ foram exportados para a Malásia, $25,1 \%$ para os Países Baixos, 7,3\% para o Japão e apenas 2,3\% para os EUA (AETAKASANAWAN, 2001). No Brasil, não existe relato da exportação de minimilho fresco (MILES e ZENS, 1998).

O consumo, assim como a produção de minimilho, que antes era maior na Ásia, atualmente está se expandindo mundialmente, especialmente na África, América do Sul e Oceania. Porém, as informações estatísticas sobre a produção e o consumo de minimilho são limitadas, porque muitos países produtores negligenciam ou não possuem essas informações (MILES e ZENS, 1998).

No Brasil, o minimilho é importado exclusivamente na forma de conservas ou enlatado. Essas conservas são reembaladas em recipientes menores, com rótulos da empresa importadora (SANTOS, 2002). Existem relatos no Brasil, assim como nos Estados Unidos e Japão, da preferência para o consumo in natura do minimilho nacional, pelo fato de esse produto não conter conservantes e outros aditivos químicos, os quais estão presentes no produto importado (MILES e ZENS,
1998). Por isso, a produção de minimilho in natura cresceu em países importadores e mais especificamente no Brasil, dando oportunidade aos produtores de processar e enlatar seus produtos para o mercado interno (MILES e ZENS, 1998; SANTOS, 2002).

No Brasil, várias cultivares de milho têm sido avaliadas com o intuito de identificar aquelas mais adaptadas às condições tropicais. Têm-se utilizado, principalmente, cultivares selecionadas de germoplasma de milho-doce e de pipoca e também, em menor escala e com grande potencial de uso, cultivares prolíficas selecionadas de milho comum (GALINAT e LIN, 1988; PEREIRA FILHO et al., 1988).

A colheita deve ser realizada pela manhã, quando a umidade das espigas é mais alta e a temperatura ambiente mais baixa, favorecendo, assim, a qualidade do produto (MILES e ZENS, 1998). O ponto ideal de colheita do minimilho é quando as espigas estão com dois a três dias de exposição dos estilos-estigma. Geralmente, são efetuadas de duas a três colheitas por planta, dependendo da cultivar e da época de semeadura (GALINAT, 1985; THAKUR e SHARMA, 1999). Após a colheita, são realizados o despalhamento e a seleção criteriosa de espigas que se enquadram em padrões comerciais, ou seja, que apresentam tamanhos variando de 4 a $12 \mathrm{~cm}$, diâmetros de 1,0 a 1,5 cm, forma cilíndrica e coloração variando de branco-pérola a amarelo-claro (KITIPRAWAT, 1989).

As espigas devem ser conservadas em local fresco e arejado, o que promove menor perda de água, impedindo a fermentação e a consequiente depreciação do produto, sendo mais recomendado o armazenamento em câmaras frias, com umidade relativa em torno de $90 \%$ e temperatura de 5 a $10^{\circ} \mathrm{C}$ (PEREIRA FILHO et al., 1998). Após três dias de armazenamento, a redução no peso em conseqüência das perdas de água na evaporação e respiração chega a, aproximadamente, 6,8\%, reduzindo também a qualidade, em razão da maturação do sabugo (LEKAGUL et al., 1981).

Na indústria, o minimilho é processado antes da comercialização utilizando os mesmos procedimentos comumente utilizados para outros legumes (CHEVAISARAKUL e PARIPATTANANONT, 1998).

No Brasil, há carência de informações e protocolos para a avaliação tecnológica desse tipo de produto. A necessidade e a importância de se aumentar os conhecimentos a respeito desse assunto motivaram a elaboração deste estudo, objetivando-se determinar a composição física e química de cultivares utilizados para a produção do minimilho. 
Foram avaliadas oito cultivares de milho, incluindo um híbrido simples, DKB 929 (grãos), seis híbridos triplos, DAS 170 (milho verde), FO 01 (silagem), CO 9621 (grãos e silagem), DO 04 (doce), Elisa (doce) e Zélia (pipoca) e uma variedade, Pipoca-estéril (minimilho).

Em 10 de fevereiro de 2001, as sementes de cada cultivar foram semeadas em 12 fileiras de $5 \mathrm{~m}$ de comprimento espaçadas de $80 \mathrm{~cm}$, na área experimental do Departamento de Agricultura, no campus da Universidade Federal de Lavras (UFLA), em Lavras-MG. A densidade de semeadura foi de 22 sementes por metro linear, e após a realização de desbaste, quando as plantas atingiram $20 \mathrm{~cm}$ de altura, a população foi ajustada para 180 mil plantas por hectare.

A adubação de semeadura foi de $400 \mathrm{~kg} \cdot \mathrm{ha}^{-1}$ da fórmula $8(\mathrm{~N}): 28\left(\mathrm{P}_{2} \mathrm{O}_{5}\right): 16\left(\mathrm{~K}_{2} \mathrm{O}\right)$. Quando as plantas apresentavam entre quatro e seis folhas, foi realizada a primeira adubação de cobertura com a aplicação de 60 kg.ha ${ }^{-1}$ de $\mathrm{N}$, utilizando-se como fonte o sulfato de amônio. A segunda adubação de cobertura foi realizada quando as plantas apresentavam entre oito e nove folhas, com a aplicação de $60 \mathrm{~kg} \cdot \mathrm{ha}^{-1} \mathrm{de} \mathrm{N}$, na forma de uréia. A colheita foi realizada três dias após a emissão dos estilos-estigma, aproximadamente aos 65 dias após a emergência das plantas. Foram efetuadas três colheitas por parcela, em intervalos de dois dias. O término da colheita ocorreu quando foi efetuada a colheita da terceira ou quarta espiga da planta. As espigas colhidas foram colocadas em sacos plásticos ou balaios próprios para colheita.

As características físicas e químicas determinadas neste trabalho incluem: a composição centesimal, que equivale aos teores de água, carboidratos, proteínas, extrato etéreo, cinzas e fibra bruta; os teores de açúcares, que equivalem aos teores de açúcares totais, redutores e não-redutores; os teores de vitamina $C$; constituintes antinutricionais, que equivalem ao teor de tanino; sólidos solúveis totais; acidez total titulável e pH. Para a determinação dessas características, foi retirada amostra contendo 10 espigas de cada cultivar. As análises do minimilho in natura foram realizadas no Laboratório de Produtos Vegetais do Departamento de Ciência dos Alimentos da UFLA. Todas as análises laboratoriais foram realizadas com três repetições e o delineamento adotado foi o inteiramente casualizado.

As espigas de minimilho de cada amostra foram trituradas e homogeneizadas com o auxílio de miniprocessador doméstico. Parte do homogenato foi imediatamente utilizada para a avaliação do $\mathrm{pH}$, da acidez ti- tulável, dos sólidos solúveis e dos compostos fenólicos, e parte foi acondicionada a $-18^{\circ} \mathrm{C}$, por 24 horas, para avaliação dos açúcares e da vitamina $\mathrm{C}$. O restante foi acondicionado em placa de Petri a $-18^{\circ} \mathrm{C}$, por 24 horas, imediatamente desidratada no liofilizador a $-40^{\circ} \mathrm{C}$, por 48 horas, para determinação da umidade e, posteriormente, para as análises de determinação de proteína, de extrato etéreo, de cinzas e de fibra bruta.

$\mathrm{O}$ grau de umidade foi determinado a frio pelo processo de liofilização a vácuo, a uma temperatura de $-40^{\circ} \mathrm{C}$, por um período de 48 horas, e expresso em porcentagem.

O teor de nitrogênio foi determinado pelo método micro-Kjeldahl (AOAC, 1990). A proteína bruta foi calculada utilizando-se o fator de conversão 6,25 e expressa em porcentagem.

Para o extrato etéreo, a extração foi feita no extrator tipo "Soxhlet", utilizando-se éter sulfúrico como solvente (AOAC, 1990). O teor de cinzas foi determinado pelo método gravimétrico, após incineração do material em mufla a 550-600 ${ }^{\circ} \mathrm{C}$ (AOAC, 1990).

Os teores de fibra bruta, expressos em porcentagem, foram determinados pelo método gravimétrico após extração por hidrólise ácida, segundo a metodologia descrita por Kramer e Ginkel (1952).

A partir do extrato líquido obtido por homogeneização (diluição 1:5), determinou-se o conteúdo de sólidos solúveis em refratômetro digital, modelo Atago PR-100, com compensação de temperatura automática a $25^{\circ} \mathrm{C}$, expresso em graus Brix.

$\mathrm{O} \mathrm{pH}$ foi determinado em medidor de $\mathrm{pH}$ digital, modelo portátil DM pH-2, Hanna Instruments, segundo técnica da AOAC (1990).

A acidez total titulável foi determinada por titulação (diluição 1:5) com $\mathrm{NaOH} 0,1 \mathrm{~N}$ e expressa em \% de ácido (AOAC, 1990).

Os açúcares redutores e não-redutores foram analisados pelo método de Somogyi-Nelson (SOUTHGATE, 1991). Para a determinação dos açúcares, utilizou-se uma alíquota de $5 \mathrm{~g}$ da amostra, que foi diluída em $50 \mathrm{ml}$ de água destilada. Tomaram-se $25 \mathrm{ml}$ para a hidrólise da sacarose, seguidos de alíquota de 3 $\mathrm{ml}$ para desproteinizar. Para o doseamento, utilizou-se alíquota de 2,0 $\mathrm{ml}$ do extrato desproteinizado e 1,0 ml da solução, após hidrólise da sacarose desproteinizada.

Os teores de compostos fenólicos, expressos em $\mathrm{mg} / 100 \mathrm{~g}$, foram obtidos pelo método de Swain e Hillis (1959), utilizando-se metanol $80 \%$ como extrator, e identificados de acordo com o método de Folin-Dennis (AOAC, 1990). 
O conteúdo de ácido ascórbico foi determinado pelo método colorimétrico com 2,4 dinitrofenilhidrazina, segundo Strohecker e Henning (1967). Utilizou-se, para o doseamento, 1,0 $\mathrm{ml}$ do filtrado, adicionando-se 3 $\mathrm{ml}$ de ácido oxálico $0,5 \%$.

Os teores de cada componente avaliado foram calculados pelo programa SAAL ${ }^{\circledR}$ (TOMÉ, 2001). As médias foram calculadas e associadas a seus respectivos desvios-padrão, a 5\% de probabilidade.

Os teores médios de água observados para as cultivares variaram de 90,2 (FO 01) a 94,5\% (Pipoca estéril) (Tabela 1). Esses valores estão de acordo com os relatados por Yodpet (1979), que encontrou teores médios de $89 \%$ de água no minimilho, enquanto em algumas hortaliças os teores variaram de $90,30 \%$ (couveflor) a $96,40 \%$ (pepino). Por ser composto basicamente de água, o minimilho requer cuidados nas fases de póscolheita, de armazenamento e de comercialização, para prevenir a perda de massa, um dos principais problemas enfrentados durante o armazenamento de produtos hortícolas, que tem efeitos marcantes sobre a fisiologia dos tecidos vegetais e que, em alguns casos, antecipa a maturação e a senescência de frutos tropicais, por exemplo (YANG e HOFFMANN, 1984). A relação da perda de massa com a perda de água, causa principal da deterioração, resulta não somente em perdas quantitativas, mas também na aparência (murchamento e enrugamento), nas qualidades texturais (amaciamento, perda de frescura e suculência) e na qualidade nutricional (KADER, 1992).

Os teores médios de carboidratos das cultivares variaram de 4,12 (Pipoca estéril) a 7,23\% (FO 01) (Tabela 1). Os teores de carboidratos relatados por Yodpet (1979) em algumas hortaliças, como, por exemplo, couve-flor, tomate, berinjela e pepino, foram de 6,10 , $4,10,5,70$ e $2,40 \%$, respectivamente. No minimilho, o teor de carboidratos foi de $8,20 \%$, ou seja, maior que o das hortaliças acima relatadas.

Os teores médios de proteína das cultivares variaram de 0,86 (Pipoca estéril) a 1,53 g/100g (FO 01) (Tabela 1). Esses teores são menores do que os relatados como padrão para o minimilho pelo INSTITUTE OF NUTRITION (1999), que varia de 1,60 a 1,90 $\mathrm{g} / 100 \mathrm{~g}$, e também menores do que o valor médio $(1,90$ $\mathrm{g} / 100 \mathrm{~g}$ ) encontrado por Yodpet (1979). De acordo com o mesmo autor, em $100 \mathrm{~g}$ de couve-flor fresca, o teor de proteína foi de 2,40 g e, em tomate, 1,00 g. Comparando o minimilho com o milho comum, após 25 dias da polinização, Machado (1988) encontrou valores entre 11,3 e 13,3 g de proteínas. Provavelmente, essa diferença no teor de proteína do minimilho deve-se à sua imaturidade, visto que a fertilização, responsável pela translocação de nitrogênio, ainda não ocorrera.

Na composição química do minimilho, o teor de proteína $(1,10 \%)$ é baixo e considerando-se que as necessidades diárias de um homem adulto são de $63 \mathrm{~g}$ de proteínas, apenas $1,75 \%$ dessas necessidades poderá ser suprida (INSTITUTE OF NUTRITION, 1999).

Os teores de extrato etéreo das cultivares variaram de 0,16 (Elisa e DOS 170) a 0,19 g/100 g (FO 01, Zélia, DO 04 e Pipoca estéril) (Tabela 1). Esses valores são baixos porque antes da fertilização a taxa de translocação de nutrientes para a espiga ainda é muito reduzida, quando comparada com uma espiga cujos óvulos já foram fertilizados. Esses resultados estão de acordo com os relatados por Yodpet (1979) e pelo INSTITUTE OF NUTRITION (1999), os quais indicam valor médio de $0,20 \mathrm{~g} / 100 \mathrm{~g}$ de minimilho fresco, semelhante aos teores de extrato etéreo encontrados em couve, tomate, berinjela e pepino. Em milho comum, após 25 dias da polinização, o valor encontrado por Machado (1988) foi de 4,8 g/100g.

TABELA 1 - Resultados da composição centesimal (g/100g na base seca): teores de carboidratos, umidade, proteína, extrato etéreo, cinzas e fibra bruta, de oito cultivares de milho.

\begin{tabular}{lcccccccc}
\hline Componentes & FO 01 & Zélia & DKB 929 & Elisa & DOS 170 & DO 04 & CO 9621 & Pipoca estéril \\
\hline Carboidratos & $\mathbf{7 , 2 3} \pm \mathbf{0 , 3 1}$ & $\mathbf{4 , 5 8} \pm \mathbf{0 , 3 4}$ & $\mathbf{4 , 5 9} \pm \mathbf{0 , 1 7}$ & $\mathbf{4 , 1 5} \pm \mathbf{0 , 0 7}$ & $\mathbf{6 , 3 3} \pm \mathbf{0 , 1 2}$ & $\mathbf{6 , 3 7} \pm \mathbf{0 , 1 8}$ & $\mathbf{4 , 8 6} \pm \mathbf{0 , 0 9}$ & $\mathbf{4 , 1 2} \pm 0,18$ \\
Umidade & $\mathbf{9 0 , 2 2} \pm 0,13$ & $\mathbf{9 3 , 7 7} \pm \mathbf{0 , 3 5}$ & $\mathbf{9 3 , 7 5} \pm \mathbf{0 , 3 1}$ & $\mathbf{9 4 , 4 4} \pm \mathbf{0 , 4 4}$ & $\mathbf{9 1 , 6 0} \pm \mathbf{0 , 2 1}$ & $\mathbf{9 1 , 3 3} \pm \mathbf{0 , 2 4}$ & $\mathbf{9 3 , 4 5} \pm \mathbf{0 , 4 1}$ & $\mathbf{9 4 , 4 7} \pm \mathbf{0 , 3 9}$ \\
Proteína & $\mathbf{1 , 5 3} \pm 0,11$ & $\mathbf{0 , 9 7} \pm \mathbf{0 , 0 8}$ & $\mathbf{0 , 9 8} \pm \mathbf{0 , 1 3}$ & $\mathbf{0 , 8 7} \pm \mathbf{0 , 1 0}$ & $\mathbf{1 , 2 3} \pm \mathbf{0 , 0 4}$ & $\mathbf{1 , 3 5} \pm \mathbf{0 , 0 7}$ & $\mathbf{1 , 0 3} \pm \mathbf{0 , 1 1}$ & $\mathbf{0 , 8 6} \pm \mathbf{0 , 1 2}$ \\
Extrato etéreo & $\mathbf{0 , 1 9} \pm 0,04$ & $\mathbf{0 , 1 2} \pm 0,04$ & $\mathbf{0 , 1 1} \pm \mathbf{0 , 0 3}$ & $\mathbf{0 , 0 9} \pm \mathbf{0 , 0 4}$ & $\mathbf{0 , 1 3} \pm \mathbf{0 , 0 4}$ & $\mathbf{0 , 1 6} \pm \mathbf{0 , 0 7}$ & $\mathbf{0 , 1 0} \pm 0,05$ & $\mathbf{0 , 1 1} \pm \mathbf{0 , 0 8}$ \\
Cinzas & $\mathbf{0 , 2 9} \pm 0,09$ & $\mathbf{0 , 1 8} \pm \mathbf{0 , 0 4}$ & $\mathbf{0 , 1 8} \pm \mathbf{0 , 0 7}$ & $\mathbf{0 , 1 6} \pm \mathbf{0 , 0 5}$ & $\mathbf{0 , 2 5} \pm 0,09$ & $\mathbf{0 , 2 5} \pm \mathbf{0 , 1 0}$ & $\mathbf{0 , 1 9} \pm 0,06$ & $\mathbf{0 , 1 6} \pm \mathbf{0 , 0 8}$ \\
Fibra bruta & $\mathbf{0 , 5 4} \pm 0,12$ & $\mathbf{0 , 3 8} \pm 0,10$ & $\mathbf{0 , 3 9} \pm 0,17$ & $\mathbf{0 , 2 9} \pm \mathbf{0 , 0 9}$ & $\mathbf{0 , 4 6} \pm \mathbf{0 , 1 1}$ & $\mathbf{0 , 5 4} \pm \mathbf{0 , 1 3}$ & $\mathbf{0 , 3 7} \pm \mathbf{0 , 0 9}$ & $\mathbf{0 , 2 8} \pm \mathbf{0 , 1 1}$ \\
\hline
\end{tabular}

Ciênc. agrotec., Lavras. V.27, n.6, p.1419-1425, nov./dez., 2003 
Os teores de cinzas variaram de 0,16 (Elisa e Pipoca estéril) a $0,29 \mathrm{~g} / 100 \mathrm{~g}$ (FO 01) (Tabela 1). Já o teor de cinzas encontrado em milho comum, após 25 dias da polinização, variou de 1,5 a 2,2 g (MACHADO, 1988).

Os teores de fibra bruta das cultivares variaram de 0,29 (Elisa) a 0,54 g/100 g (FO 01 e DO 04) (Tabela 1). O INSTITUTE OF NUTRITION (1999) sugere valores de fibra de $1,1 \mathrm{~g}$. Yodpet (1979) encontrou 0,6 g em 100 g de minimilho. Na composição química do minimilho, o teor de fibras, por exemplo, supre apenas $1,50 \%$ das necessidades humanas, que são de 20 a $35 \mathrm{~g} / \mathrm{dia}$ de fibra alimentar (INSTITUTE OF NUTRITION, 1999).

Os açúcares são muito importantes para o sabor do minimilho e variam de acordo com a cultivar e as condições climáticas. No milho comum, a concentração de açúcares totais aumenta durante o desenvolvimento das espigas, até o início do amadurecimento. A variação do teor de açúcares totais foi de 1,24 (Pipoca estéril) a 2,12 g/100 g (FO 01) (Tabela 2). Os valores de açúcares redutores (glicose) variaram de 0,74 (Pipoca estéril) a 1,28 g/100 g (FO 01) e, para os nãoredutores (sacarose), a variação foi de 0,50 (Elisa) a 0,85 g/100 g (FO 01). Bar-Zur e Shaffer (1993) determinaram teores de açúcares totais de 2,0 a 3,0 g/100 g. Em açúcares redutores, glicose e frutose, os teores foram de 1,0 a 1,5 g/100 g, mencionando-se que a sacarose encontra-se em pequenas quantidades no ponto de colheita de minimilho.

A variação no teor de ácido ascórbico (vitamina C) foi de 9,58 (DO 04) a 11,5 mg/100 g (CO 9621)
(Tabela 2). Yodpet (1979) encontrou valor de 11 $\mathrm{mg} / 100 \mathrm{~g}$ de ácido ascórbico em minimilho fresco. De acordo com a tabela de composição de alimentos publicada por Franco (1998), o teor de ácido ascórbico para milho comum verde é de $16,4 \mathrm{mg} / 100 \mathrm{~g}$, e para o milho pipoca, 9,0 mg/100 g. O teor de ácido ascórbico (vitamina C) depende de muitos fatores, incluindo efeito de cultivares, estádio de maturação, condições edafoclimáticas e acidez do solo. As condições de pós-colheita e armazenamento influenciam no teor de ácido ascórbico, mesmo antes do processamento (ROIG et al., 1993).

O teor de compostos fenólicos (tanino), expresso em mg de equivalente de ácido tânico/100 g, variou de 168,5 (Elisa) a 190,27 mg/100 g (FO 01) (Tabela 2). Entre os fatores antinutricionais, os compostos fenólicos merecem atenção por serem responsáveis pela formação de cor indesejável, em razão da oxidação em meio alcalino e da formação, por meio de múltiplas pontes de hidrogênio com a cadeia polipeptídica, de um complexo proteína-tanino que impede a digestibilidade da proteína (ESPÍNDOLA, 1987). No minimilho, quando enlatado, são adicionados vinagre e substâncias salinas para diminuir o $\mathrm{pH}$, prevenindo a oxidação em meio alcalino.

Para o $\mathrm{pH}$, verificou-se uniformidade de valores, ou seja, variação de 6,42 (Zélia) a 6,95 (Elisa). A variação nos teores de sólidos solúveis totais foi de 6,50 (DO 04) a 10,50\% (FO 01 e DOS 170). A variação da acidez total titulável foi de 1,50 (FO 01) a 3,50\% (DOS 170 e DO 04) (Tabela 2).

TABELA 2 - Teores de sólidos solúveis totais - SST (\%), da acidez total titulável - ATT (\%), do pH, dos teores de açúcares (totais, redutores e não-redutores - g/100 g), da vitamina C (mg/100 g) e do tanino (mg/100 g), com base na matéria fresca, de oito cultivares de milho.

\begin{tabular}{|c|c|c|c|c|c|c|c|c|}
\hline Teores & FO 01 & Zélia & DKB 929 & Elisa & DOS 170 & DO 04 & CO 9621 & $\begin{array}{l}\text { Pipoca } \\
\text { estéril }\end{array}$ \\
\hline SST & $10,5 \pm 0,94$ & $9,50 \pm 0,65$ & $8,50 \pm 0,56$ & $7,50 \pm 1,05$ & $10,50 \pm 0,60$ & $6,50 \pm 1,10$ & $9,50 \pm 0,74$ & $8,50 \pm 1,05$ \\
\hline ATT & $1,50 \pm 0,13$ & $2,00 \pm 0,26$ & $2,50 \pm 0,26$ & $3,00 \pm 0,39$ & $3,50 \pm 0,28$ & $3,50 \pm 0,27$ & $2,50 \pm 0,21$ & $2,50 \pm 0,28$ \\
\hline $\mathrm{pH}$ & $6,83 \pm 0,41$ & $6,42 \pm 0,28$ & $6,82 \pm 0,40$ & $6,95 \pm 0,40$ & $6,57 \pm 0,48$ & $6,85 \pm 0,41$ & $6,82 \pm 0,34$ & $6,62 \pm 0,38$ \\
\hline Glicose & $1,28 \pm 0,19$ & $0,84 \pm 0,21$ & $0,83 \pm 0,23$ & $0,76 \pm 0,21$ & $1,10 \pm 0,21$ & $1,18 \pm 0,25$ & $0,88 \pm 0,20$ & $0,74 \pm 0,15$ \\
\hline Sacarose & $0,85 \pm 0,22$ & $0,57 \pm 0,24$ & $0,58 \pm 0,24$ & $0,50 \pm 0,22$ & $0,82 \pm 0,19$ & $0,78 \pm 0,25$ & $0,59 \pm 0,17$ & $0,50 \pm 0,16$ \\
\hline Totais & $2,12 \pm 0,20$ & $1,41 \pm 0,12$ & $1,41 \pm 0,18$ & $1,26 \pm 0,18$ & $1,92 \pm 0,23$ & $1,96 \pm 0,20$ & $1,47 \pm 0,20$ & $1,24 \pm 0,16$ \\
\hline Vit. C & $11,54 \pm 0,31$ & $11,00 \pm 0,87$ & $10,40 \pm 0,16$ & $10,20 \pm 0,15$ & $10,10 \pm 0,16$ & $9,58 \pm 0,35$ & $11,50 \pm 0,21$ & $11,00 \pm 0,47$ \\
\hline Tanino & $190,27 \pm 12,45$ & $\begin{array}{c}188,98 \pm \\
11,91\end{array}$ & $172,86 \pm 12,03$ & $168,5 \pm 12,07$ & $171,87 \pm 12$ & $175,86 \pm 11,98$ & $171,73 \pm 15,03$ & $\begin{array}{c}180,27 \pm \\
12,04\end{array}$ \\
\hline
\end{tabular}


Com base nos resultados obtidos neste trabalho, conclui-se que:

O minimilho é composto basicamente de água, com teores acima de $90 \%$, em média;

Os valores de umidade, carboidratos, proteína, açúcares e vitaminas das cultivares analisadas, apresentaram-se similares aos comumente relatados na literatura, porém ocorre variação da composição física e química em função da cultivar.

\section{REFERÊNCIAS BIBLIOGRÁFICAS}

AETAKASANAWAN, C. Baby corn. In: HALLAUER, A. R. (Ed.). Specialty corns. 2. ed. Iowa: CRC, 2001. v. 2, cap. 9, p. 275-293.

ASSOCIATION OF OFFICIAL AND AGRICULTURAL CHEMISTS. Official methods of analysis of the association. 15. ed. Washington: Board, 1990. v. 1-2.

BAR-ZUR, A.; SCHAFFER, A. Size and carbohydrate content of ears of baby corn in relation to endosperm type (su, su, se, sh2). Journal American Society Horticultural Sciences, Alexandria, v. 118, n. 1, p. 141-144, 1993.

CHEVA-ISARAKUL, B.; PARIPATTANANONT, T. The nutritive value of fresh baby corn waste. In: Ruminant feeding systems utilizing fibrous agricultural residues: 1987. Canberra: International Development Program of Australian Universities and Colleges, 1998. 151 p.

ESPÍNDOLA, F. S. Fracionamento dos vegetais verdes e obtenção de concentrados protéicos de folhas (CPF) para suplementação de alimentos e ração animal, com aproveitamento dos subprodutos. 1987. 130 f. Monografia (Especialização) - Universidade Federal de Uberlândia, Centro de Ciências Biomédicas, Uberlândia, 1987.

FRANCO, G. Tabela de composição química dos alimentos. 7. ed. Rio de Janeiro: Atheneu, 1998. 145 p.

GALINAT, W. C. Whole ear baby corn a new way to eat corn. Proceeding Northeast Corn Improvement Conference, Seatlle, v. 40, p. 22-27, 1985.

GALINAT, W. C.; LIN, B. Y. Baby corn: production in Taiwan and future outlook for production in the United States. Economic Botany, New York, v. 42, n. 1, p. 132-134, Jan./Mar. 1988.
INSTITUTE OF NUTRITION. Thai food composition tables. 1999. Disponível em: 〈http://agsyst.wsu.edu>. Acesso em: 12 jan. 2002.

KADER, A. A. Postharvest technology of horticultural crops. California: University of California, 1992. 296 p.

KAMER, J. H. van de; GINKEL, L. van. Rapid determination of crude fiber in cereals. Cereal Chemistry, Saint Paul, v. 29, n. 4, p. 239-251, Apr. 1952.

KITIPRAWAT, S. Other aspects of the economy. Bangkok Bank Monthly Review, Bangkok, p. 450453, Nov. 1989.

LEKAGUL, T.; PERNMAMKHONG, S.; CHUTKAEW, C.; BENJASIL, V. Field corn variety for young ear corn production. National Corn and Sorghum Program Annual Report, Bangkok, v. 13, p. 201-205, 1981.

MACHADO, M. C. M. da S. T. Estudo da composição química, características agronômicas e sensoriais de uma nova cultivar de milho, com endosperma triplo mutante sugary-opaque 2-waxy. 1988. 114 f. Dissertação (Mestrado em Ciência dos Alimentos) - Universidade Estadual de Campinas, Campinas, 1988.

MILES, C.; ZENS, L. The web of science. Washington: Washington State University, 1998. Disponível em: <http://agsyst.wsu.edu>. Acesso em: 27 out. 2001.

PEREIRA FILHO, I. A.; GAMA, E. E. G.; FURTADO, A. A. L. A produção do minimilho. In: EMPRESA BRASILEIRA DE PESQUISA AGROPECUÁRIA. Centro Nacional de Pesquisa de Milho e Sorgo. Sete Lagoas, 1988. 4 p. (Comunicado Técnico, 7).

ROIG, M. G.; RIVERA, Z. S.; KENNEDY, J. F. Lascorbic acid: an overview. International Journal of Food Science and Nutrition, Oxford, v. 44, n. 1, p. 5972, May 1993.

SANTOS, M. R. Biblioteca virtual [mensagem pessoal]. Mensagem recebida por<saporibr@terra.com.br> em 8 jan. 2002.

SOUTHGATE, D. A. T. Determination of foods carbohydrates. London: Elsevier Apllied Science, 1991. 232 p. 
STROHECKER, R.; HENNING, H. M. Análises de vitaminas: métodos comprovados. Madrid: Paz Montalvo, 1967. $428 \mathrm{p}$.

SWAIN, T.; HILLIS, W. G. The fenolic constituents of Prunus domestica. The quantitative analysis of phenolic constituints. Journal of the Science of Food and Agriculture, London, v. 10, n. 1, p. 63-68, Jan. 1959.

THAKUR, D. R.; SHARMA, V. Effect of varieties rates of nitrogen and its schedule of aplication in baby corn (Zea mays L.). Indian Journal of Agricultural Sciences, New Delhi, v. 62, n. 2, p. 93-95, Feb. 1999.
TOMÉ, P. H. F. Sistema de análise de alimentos. [S.1.: s.n.], 2001.

YANG, S. F.; HOFFMANN, N. E. Ethylene biosynthesis and its regulation in higher plants. Annual Review Plant Phisiology, Palo Alto, v. 35, p. 155-189, 1984.

YODPET, C. Studies on sweet corn as potential young cob corn (Zea mays, L). 1979. Thesis (Doctor) - University of the Philippines, Los Banos, 1979. 